Habou RABIOU ${ }^{1}$

Abdoulaye Diouf ${ }^{2}$

Babou André BATIONO3

Kossi Novinyo SEgLA 4

Kossi AdjonOU 4

Adzo Dzifa KoKUtse 4

Raoufou RADJI ${ }^{4}$

Kouami KoKou 4

Ali MAHAMANE ${ }^{1,5}$

Mahamane SAADOU 5

${ }^{1}$ Université de Diffa

Faculté des Sciences

Agronomiques (FSA)

BP 78, Diffa

Niger

2 Université Dan Dicko Dankoulodo de Maradi

Faculté d'Agronomie et des Sciences de l'Environnement BP 465, Maradi

Niger

${ }^{3}$ Institut de l'environnement et de recherches agricoles (INERA) 04 BP 8645

Ouagadougou 04

Burkina Faso

4 Université de Lomé Faculté des Sciences Laboratoire de botanique et écologie végétale BP 1515, Lomé

Togo

5 Université Abdou Moumouni Faculté des Sciences

et Techniques

Laboratoire Garba Mounkaila Département de Biologie BP 10662, Niamey Niger

\section{Structure des peuplements naturels de Pterocarpus erinaceus Poir. dans le domaine soudanien, au Niger et au Burkina Faso}

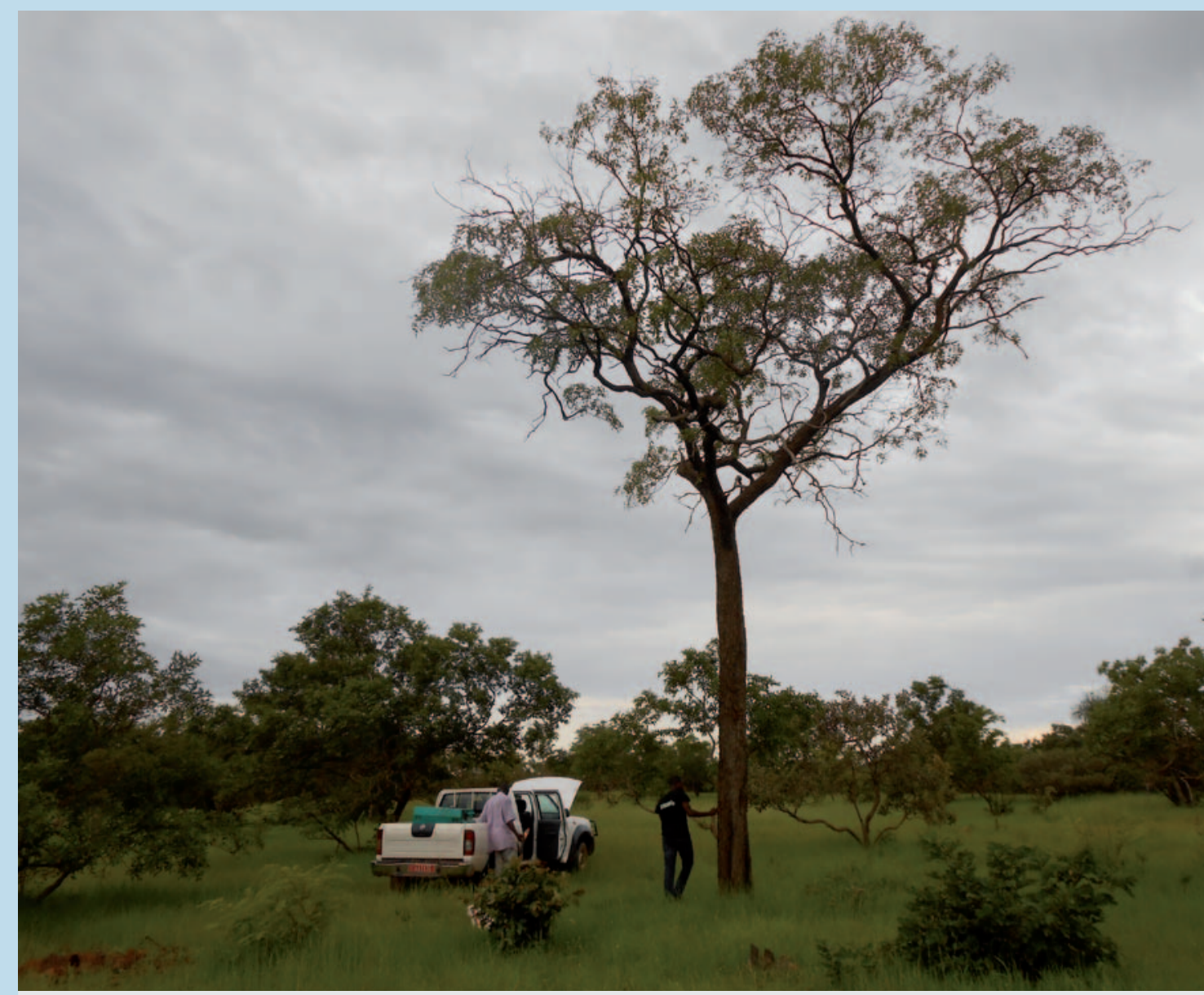

Photo 1.

Pied de Pterocarpus erinaceus.

Photo H. Rabiou, 2014. 


\section{RÉSUMÉ}

\section{STRUCTURE DES PEUPLEMENTS NATURELS DE PTEROCARPUS ERINA- CEUS POIR. DANS LE DOMAINE SOUDA- NIEN, AU NIGER ET AU BURKINA FASO}

Pterocarpus erinaceus Poir. est une espèce endémique des zones sahélo-soudanienne et soudano-guinéenne, recherchée pour son bois, son fourrage et son intérêt médicinal. Ces usages se traduisent par une mutilation répétée des individus, entraînant une dégradation sensible des peuplements naturels. Les structures démographiques de $P$. erinaceus selon les zones pédoclimatiques et l'effet des principales variables environnementales et de l'intensité d'exploitation sur ses effectifs ont été évalués au Niger et au Burkina Faso. Des mesures dendrométriques (diamètre, hauteur totale, hauteur du fût, diamètres perpendiculaires du houppier) sur les individus d'un diamètre supérieur ou égal à $5 \mathrm{~cm}$ à 1,30 $\mathrm{m}$ du sol ont été effectuées dans huit forêts. Dans chacune, les mesures ont été conduites selon deux transects perpendi-culaires. La densité de $P$. erinaceus augmente de manière significative $(P<0,003)$ de la zone sahélo-soudanienne à la zone soudano-guinéenne : 1,2 $\pm 0,7$ $\mathrm{pied} / \mathrm{ha}$ dans la zone sahélo-soudanienne ; $2,6 \pm 2,2$ pieds/ha dans la zone nord-soudanienne ; 6,1 $\pm 4,6$ pieds/ha dans la zone sud-soudanienne; $15,0 \pm 1,1$ pieds/ha dans la zone soudano-guinéenne. L'analyse des structures en diamètre et en hauteur révèle une augmentation de la fréquence des individus jeunes suivant le gradient pluviométrique. Par contre, l'intensité de l'exploitation est plus élevée dans les zones les moins arrosées, où l'espèce s'avère menacée.

Mots-clés : Pterocarpus erinaceus Poir., caractéristiques dendrométriques, structure du peuplement, pédoclimat, Burkina Faso, Niger, zone sahélienne, zone soudanienne.

\section{ABSTRACT}

\section{STRUCTURE OF NATURAL PTEROCAR- PUS ERINACEUS POIR. POPULATIONS IN THE SUDANIAN DOMAIN IN NIGER AND BURKINA FASO}

Pterocarpus erinaceus Poir. is endemic to the Sahelo-Sudanian and Sudano-Guinean zones and much sought after for its wood, fodder and medicinal properties. As a result, individual trees are repeatedly mutilated, which is causing serious damage to the natural tree stands. This study assessed the population structure of $P$. erinaceus in the different pedoclimatic zones and the effects on tree numbers of the main environmental variables and intensity of use in Niger and Burkina Faso. Individual trees with a diameter of $5 \mathrm{~cm}$ or more were measured (diameter, total height, height of the bole, perpendicular diameters of the crown) at a height of 1.30 $m$ above the ground in eigth forest formations. In each, the dendrometric measurements were made along two perpendicular transects. The density of $P$. erinaceus increases significantly $(P<0.003)$ from the Sahelo-Sudanian to the Sudano-Guinean zone: $1.2 \pm 0.7$ stems/ha in the Sahelo-Sudanian zone; $2.6 \pm 2.2$ stems/ha in the northern Sudanian zone; $6.1 \pm 4.6$ stems/ha in the southern Sudanian zone and $15.0 \pm 1.1 \mathrm{stems} / \mathrm{ha}$ in the SudanoGuinean zone. Our analysis of the stands by diameter and height shows an increasing frequency of young individuals along the pluviometric gradient. However, use intensity is greatest in the driest zones, where the species is under threat.

Keywords: Pterocarpus erinaceus Poir., dendrometric characteristics, population structure, pedoclimate, Burkina Faso, Niger, Sahelian zone, Sudanian zone.

\section{ESTRUCTURA DE LOS RODALES NATU- RALES DE PTEROCARPUS ERINACEUS POIR. EN EL DOMINIO SUDANÉS, EN NÍGER Y BURKINA FASO}

Pterocarpus erinaceus Poir. es una especie endémica de las zonas climáticas saheliano-sudanesa y sudano-guineana, apreciada por su madera, forraje e interés medicinal. Dichos usos conllevan una reiterada mutilación de los individuos, provocando una sensible degradación de los rodales naturales. Se evaluaron las estructuras demográficas de $P$. erinaceus en Níger y Burkina Faso según las zonas edafoclimáticas, el efecto de las principales variables ambientales y la intensidad de la explotación de su población. Se efectuaron mediciones dendrométricas (diámetro, altura total, altura de fuste y diámetros perpendiculares de la copa) en 8 bosques, en individuos de diámetro superior o igual a $5 \mathrm{~cm}$ a $1.30 \mathrm{~m}$ del suelo. En cada bosque, las mediciones se realizaron siguiendo dos transectos perpendiculares. La densidad de P. erinaceus se incrementa de modo significativo $(P<0,003)$ de la zona sahelianosudanesa a la sudano-guineana: $1.2 \pm 0.7$ $\mathrm{pies} / \mathrm{ha}$ en la zona saheliano-sudanesa; $2.6 \pm 2.2 \mathrm{pies} / \mathrm{ha}$ en la zona norsudanesa; $6.1 \pm 4.6 \mathrm{pies} / \mathrm{ha}$ en la zona sursudanesa y $15.0 \pm 1.1 \mathrm{pies} / \mathrm{ha}$ en la zona sudanoguineana. El análisis de las estructuras en diámetro y altura refleja un aumento de la frecuencia de individuos jóvenes a lo largo del gradiente pluviométrico. La intensidad de explotación, sin embargo, es mayor en las zonas con menos lluvias, allí donde esta especie está amenazada.

Palabras clave: Pterocarpus erinaceus Poir., características dendrométricas, estructura del rodal, edafoclima, Burkina Faso, Níger, zone saheliana, zona sudanesa. 


\section{Introduction}

L'accès, l'exploitation et la gestion durable des ressources végétales représentent des enjeux majeurs pour les populations rurales en Afrique de l'Ouest, avec des situations contrastées suivant les potentialités agro-écologiques (Drabo et al., 2003). La récurrence des déficits pluviométriques des dernières décennies a causé la baisse d'une production vivrière essentiellement basée sur la culture pluviale. Les écosystèmes naturels font dès lors l'objet d'un surcroît d'exploitation, par une population recherchant des compléments alimentaires et des sources de revenus, mais aussi d'une forte pression pastorale (Sawadogo, 2011).

Pterocarpus erinaceus Poir., espèce endémique des zones sahélo-soudanienne et soudano-guinéenne, figure parmi les espèces qui sont de ce fait les plus exploitées (Ouédraogo et al., 2006 ; Adjonou et al., 2010 ) (photo 1). Cette espèce occupe les savanes arborées semi-arides à subhumides des régions à pluviométrie annuelle comprise entre 600 et $1200 \mathrm{~mm}$, avec une température moyenne annuelle de $32{ }^{\circ} \mathrm{C}$. Elle peut survivre aux feux de brousse annuels (Arbonnier, 2004).

Cette espèce est très recherchée par les artisans burkinabè pour la confection d'instruments de musique tels que le balafon et le djembé (Ouédraogo et al., 2006) (photos 2 et 3 ). Les individus présents dans les champs, les jachères ou les forêts classées sont fréquemment émondés pour alimenter le bétail. Leur feuillage est vendu comme fourrage dans les centres urbains, au Burkina Faso, au Niger et au Mali. Au Niger, en particulier, l'espèce est utilisée en période de soudure (avril à juin) comme unique source de matière fraîche. L'exploitation porte généralement sur les jeunes feuilles et les fleurs. Les usages en médecine traditionnelle sont nombreux (Ouédraogo et al., 2011).

Cette forte pression, conjuguée au changement climatique, à la faible production semencière et à la croissance lente des plants de $P$. erinaceus, fait de cet arbre l'un des plus menacés de la région considérée. Il a de fait localement disparu dans plusieurs sites du Burkina Faso et du Niger (Sokpon et al., 2006) et, dans ce dernier pays qui recouvre la limite septentrionale de son aire de distribution (Arbonnier, 2004), les peuplements régressent et tendent vers l'extinction.

Plusieurs études ont porté sur la dendrométrie de cette espèce en Afrique de l'Ouest (Glele et al., 2008 ; Adjonou et al., 2010 ; Nacoulma, 2012 ), mettant en évidence une diversité des peuplements selon les types d'occupation des sols et le climat (Henry et al., 2011). Celle que nous avons conduite en complément visait à préciser les structures démographiques des peuplements de $P$. erinaceus selon les zones agro-écologiques, et à déterminer l'effet des facteurs écologiques et de l'intensité de l'exploitation sur leurs effectifs (photos 4 et 5 ).

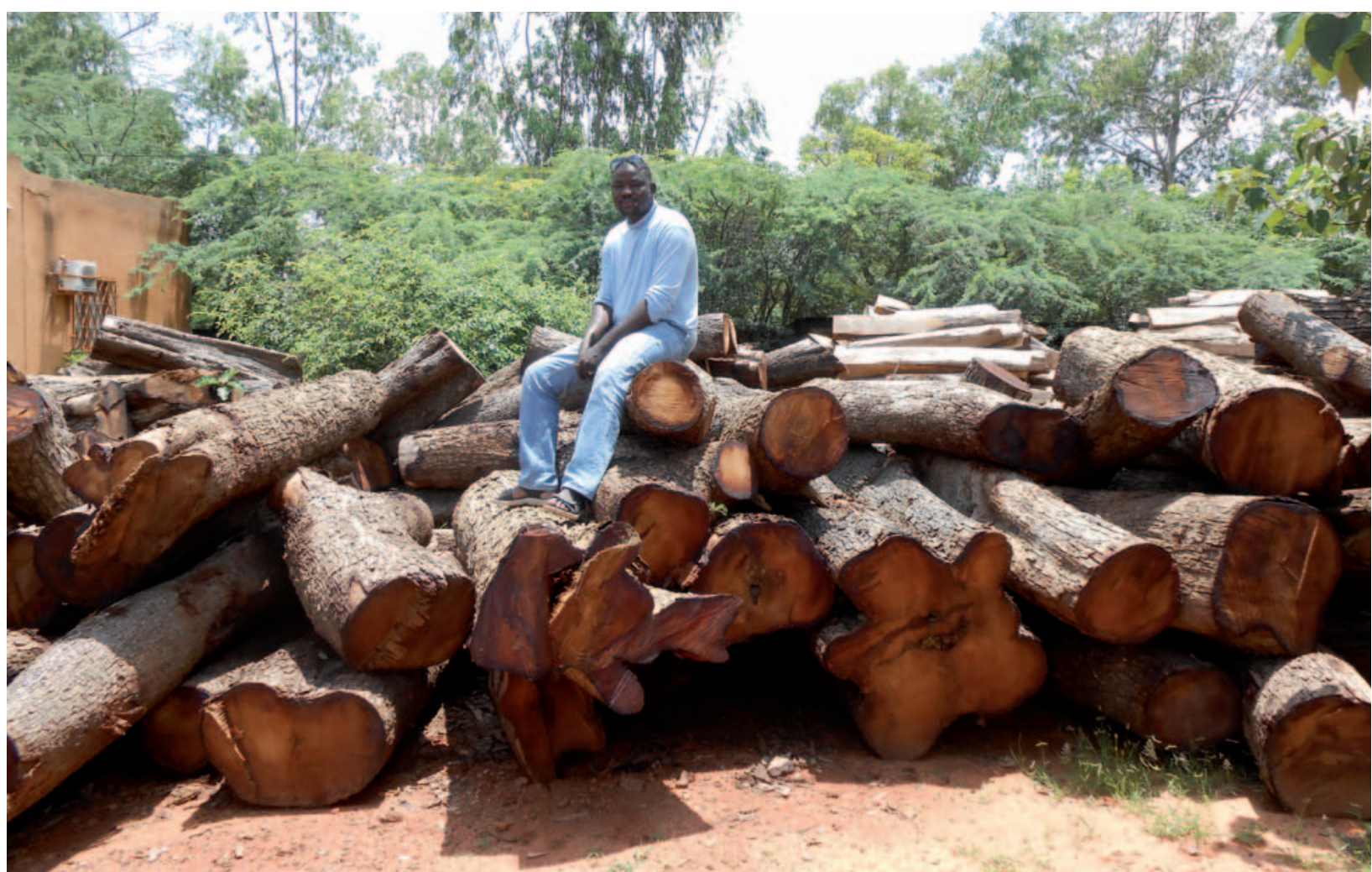

Photo 2.

Chargement de Pterocarpus erinaceus au Burkina Faso (Banfora).

Photo H. Rabiou, 2014. 


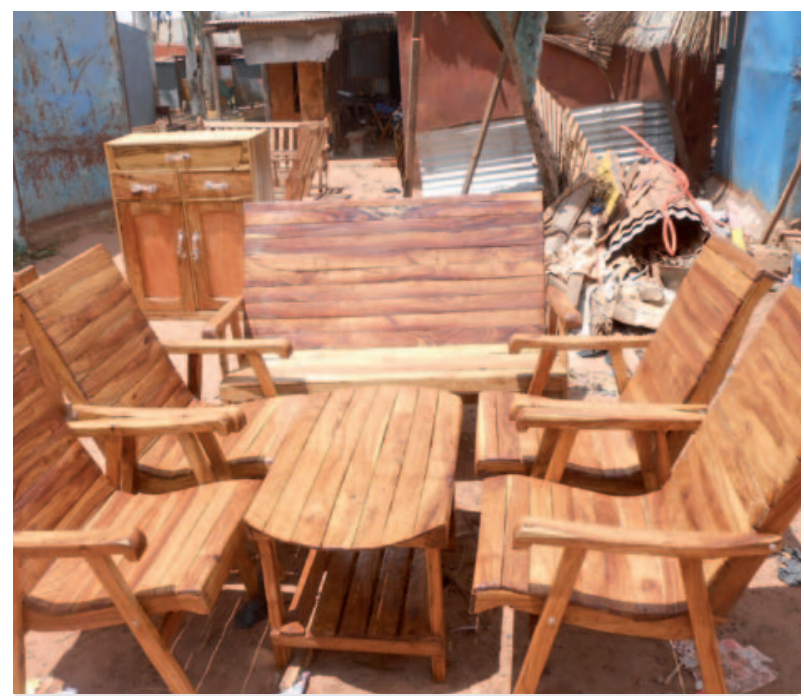

Photo 3.

Chaises de confection locale en bois de Pterocarpus erinaceus. Photo H. Rabiou, 2014.

\section{Méthodologie}

\section{Zone d'étude}

La carte des aires de distribution de $P$. erinaceus, dressée par Arbonnier (2004), a servi de base au plan d'échantillonnage de l'étude. Elle distingue quatre zones écologiques selon les caractéristiques climatiques du milieu. Dans chacune des zones, des forêts ont été choisies comme sites représentatifs afin de diagnostiquer la variabilité écologique des peuplements naturels de $P$. erinaceus. Pour mettre en évidence l'effet des conditions climatiques sur la régénération de l'espèce, deux forêts dépourvues de toute pression humaine (Parc W et réserve partielle de faune de ComoéLéraba) ont été retenues dans les deux zones écologiques extrêmes de la zone d'étude (zone sahélo-soudanienne au nord et zone soudano-guinéenne au sud). D'autres critères comme la proximité des grands centres urbains ont été également retenus afin de mettre en évidence la variabilité du degré d'exploitation de l'espèce étudiée.

\section{Zone sahélo-soudanienne $(500-700 \mathrm{~mm})$}

Dans la zone sahélo-soudanienne, deux sites ont été investigués : la portion nigérienne du Parc régional du W (220 000 ha), située entre $11^{\circ} 00^{\prime}$ et $12^{\circ} 35^{\prime}$ de latitude Nord et entre $2^{\circ} \mathrm{OO}$ et $3^{\circ} 50^{\prime}$ de longitude Est, avec une pluviométrie moyenne annuelle de $704 \pm 101 \mathrm{~mm}$ (figure 1); la réserve de faune de Tamou ( 76 ooo ha) au Niger, située entre $12^{\circ} 28^{\prime}$ et $12^{\circ} 50^{\prime}$ de latitude Nord et entre $2^{\circ} 06^{\prime}$ et $2^{\circ} 24^{\prime}$ de longitude Est, avec une pluviométrie moyenne annuelle de $606 \pm 99 \mathrm{~mm}$. Les températures moyennes annuelles sont respectivement de $29^{\circ} \mathrm{C}$ et $31^{\circ} \mathrm{C}$. La végétation est une mosaïque de forêts claires, de savanes arborées, de savanes arbustives et de savanes herbeuses.

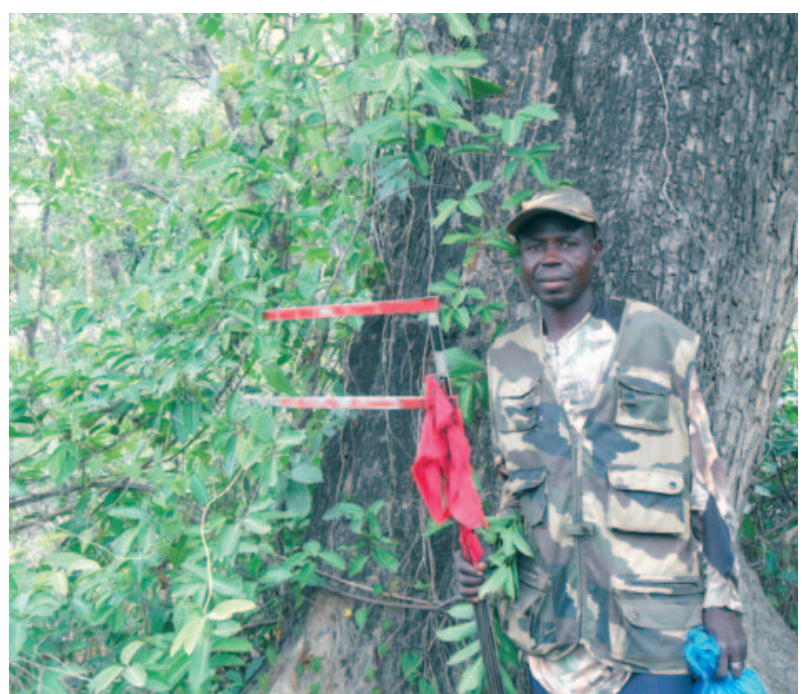

Photo 4 .

Arbre de Pterocarpus erinaceus de plus d'un mètre de diamètre. Photo H. Rabiou, 2014.

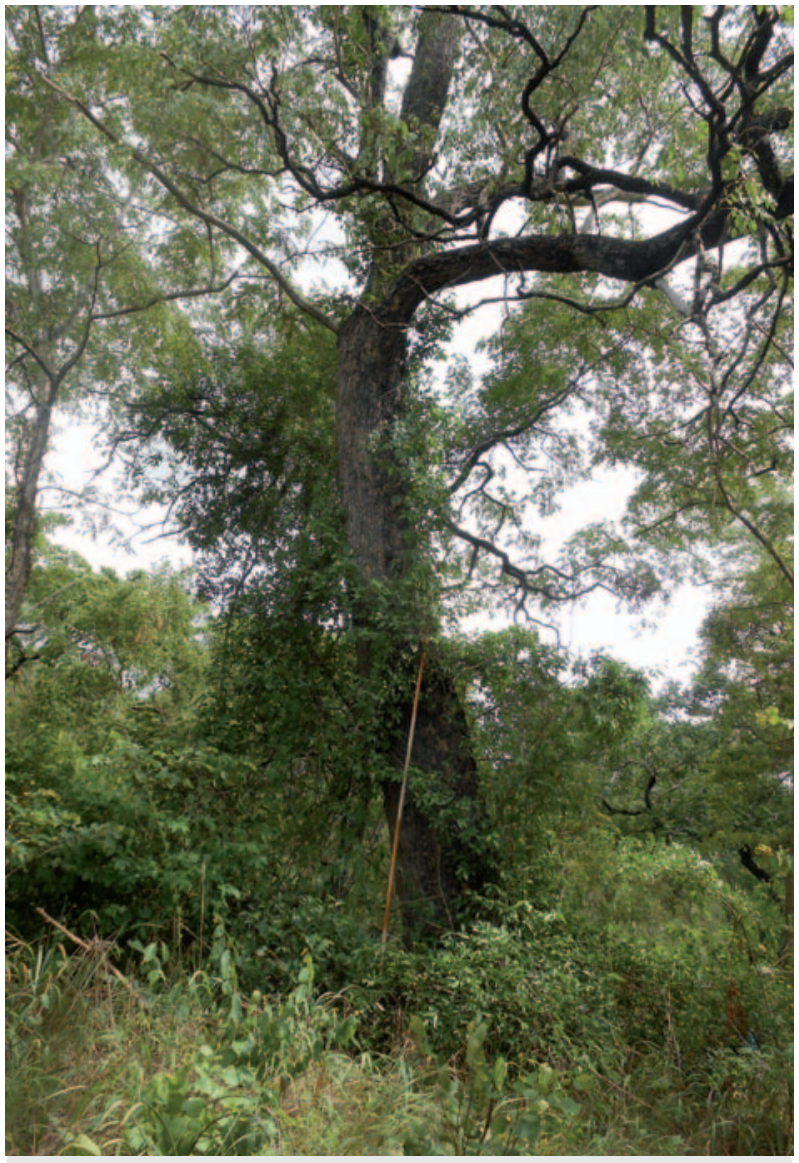

Photo 5.

Arbre de Pterocarpus erinaceus au Burkina Faso. Photo H. Rabiou, 2014. 


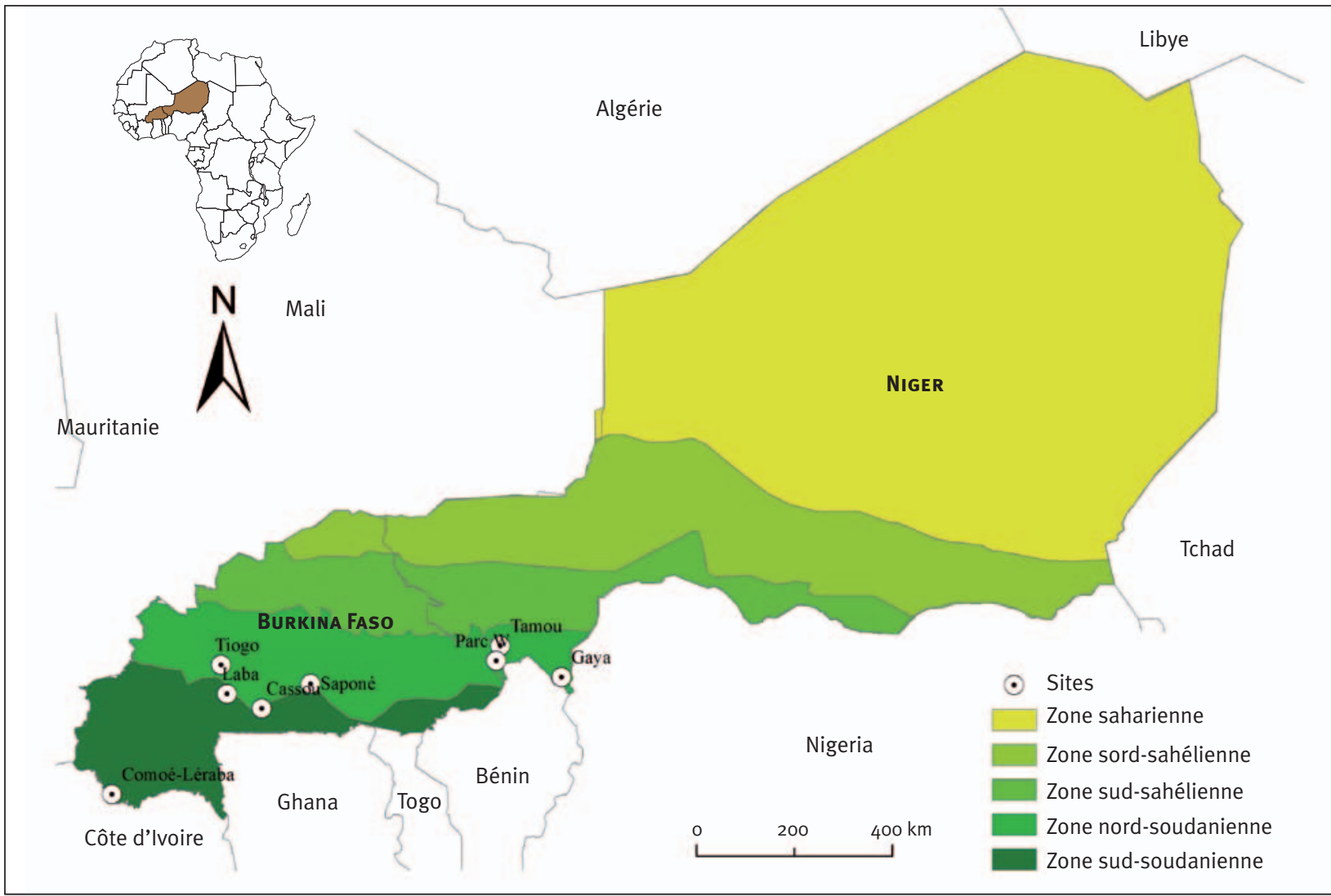

Figure 1.

Situation géographique des zones d'étude.

\section{Zone nord-soudanienne (700-900 mm)}

Trois sites ont été retenus dans cette zone (figure 1) : les forêts de Tiogo (30 389 ha) au Burkina Faso, situées entre $12^{\circ} 11^{\prime}$ et $12^{\circ} 24^{\prime}$ de latitude Nord et entre $2^{\circ} 39^{\prime}$ et $2^{\circ} 52^{\prime}$ de longitude Ouest, caractérisées par une pluviométrie moyenne annuelle de $827 \pm 169 \mathrm{~mm}$; la forêt de Saponé (100 ha) au Burkina Faso, située entre $12^{\circ} 07^{\prime}$ et $12^{\circ} 08^{\prime}$ ' de latitude Nord et entre $1^{\circ} 33^{\prime}$ et $1^{\circ} 34^{\prime}$ de longitude Ouest, avec une pluviométrie moyenne annuelle de $806 \pm 102 \mathrm{~mm}$; la forêt de Gaya (9 970 ha) au Niger, s'étendant entre $11^{\circ} 52^{\prime}$ et $11^{\circ} 58^{\prime}$ de latitude Nord et entre $3^{\circ} 20^{\prime}$ et $3^{\circ} 26^{\prime}$ de longitude Est, et caractérisée par une pluviométrie moyenne annuelle de $740 \pm 119 \mathrm{~mm}$. Les températures moyennes annuelles sont respectivement de $24^{\circ} \mathrm{C}, 26^{\circ} \mathrm{C}$ et $33^{\circ} \mathrm{C}$. Les principaux types de végétation rencontrés dans cette zone sont la savane arborée, la savane arbustive et la savane herbeuse, qui s'étendent sur des sols superficiels et gravillonnaires.

\section{Zone sud-soudanienne (900-1 $100 \mathrm{~mm})$}

Deux sites ont été inventoriés au sein de la zone sudsoudanienne (figure 1) : la forêt de Cassou (29 515 ha) au Burkina Faso, située entre $11^{\circ} 44^{\prime}$ et $11^{\circ} 21^{\prime}$ de latitude Nord et entre $2^{\circ} 07^{\prime}$ et $1^{\circ} 44^{\prime}$ de longitude Ouest, avec une pluviométrie moyenne annuelle de $984 \pm 103 \mathrm{~mm}$; la forêt de Laba
(18 501 ha) au Burkina Faso, localisée entre $11^{\circ} 48^{\prime}$ et $11^{\circ} 39^{\prime}$ de latitude Nord et entre $2^{\circ} 44^{\prime}$ et $2^{\circ} 36^{\prime}$ de longitude Ouest, avec une pluviométrie moyenne de $907 \pm 157 \mathrm{~mm}$. La température moyenne annuelle est de $24^{\circ} \mathrm{C}$ avec une amplitude thermique de $15{ }^{\circ} \mathrm{C}$. Le paysage de ces trois sites est constitué d'une mosaïque de savanes boisées à arborées et de forêts claires imbriquées.

\section{Zone soudano-guinéenne (1 100-1 $300 \mathrm{~mm})$}

Dans la zone soudano-guinéenne (figure 1), seule la réserve partielle de faune de Comoé-Léraba au Burkina Faso, située entre $9^{\circ} 39^{\prime}$ et $10^{\circ} 00^{\prime}$ de latitude Nord et entre $4^{\circ} 25^{\prime}$ et $4^{\circ} 59^{\prime}$ de longitude Ouest, a été investiguée. La superficie de cette forêt est de 125000 ha et se caractérise par une pluviométrie moyenne annuelle de $1115 \mathrm{~mm}$ et une température moyenne annuelle de $27^{\circ} \mathrm{C}$. La végétation se compose de savanes, de forêts claires et de galeries forestières.

\section{Collecte des données}

La faible densité de $P$. erinaceus dans les formations naturelles des sites retenus a conduit au choix d'un échantillonnage selon des transects en bande. Dans chaque site, deux transects perpendiculaires (directions Nord-Sud et 
Ouest-Est) d'environ $200 \mathrm{~m}$ de largeur et de longueur variable selon les sites (tableau I) ont guidé la caractérisation des peuplements (figure 2). Cette approche a permis de prendre en compte l'hétérogénéité structurale des peuplements et d'inventorier suffisamment d'individus pour estimer la densité et la structure démographique de manière fiable.

Dans chaque transect, un azimut a été fixé à l'aide d'un récepteur GPS (Global Positioning System). Cet azimut était suivi lors de l'inventaire, et tous les individus de $P$. erinaceus rencontrés ayant un diamètre à 1,30 m supérieur ou égal à $5 \mathrm{~cm}$ ont été mesurés. Afin de ne pas dépasser $100 \mathrm{~m}$ de part et d'autre suivant l'azimut, la distance de l'individu par rapport à cet azimut a été estimée à l'aide d'un télémètre laser. Les mesures des individus ont porté sur le diamètre à 1,30 m du sol, la hauteur totale, la hauteur du fût, et deux diamètres perpendiculaires du houppier. Les traces d'exploitation éventuelles (émondage, écorçage) et la texture de sol déterminée selon une approche tactile ont été notées (Ambouta, 1984). Les distances parcourues et les superficies des transects en bande couvertes sont reportées sur le tableau I.

Tableau I.

Situation et caractéristiques des transects parcourus.

\begin{tabular}{|l|c|c|c|c|}
\hline \multirow{2}{*}{$\begin{array}{l}\text { Zones } \\
\text { écologiques }\end{array}$} & Site & Pays & $\begin{array}{c}\text { Longueur totale } \\
\text { des transects } \\
(\mathbf{k m})\end{array}$ & $\begin{array}{c}\text { Superficie } \\
\text { couverte } \\
\text { (ha) }\end{array}$ \\
\hline \multirow{2}{*}{$\begin{array}{l}\text { Sahélo- } \\
\text { soudanienne }\end{array}$} & Tamou & Niger & 9,80 & 197,30 \\
\cline { 2 - 5 } & PW & Niger & 6,40 & 128,02 \\
\hline \multirow{2}{*}{$\begin{array}{l}\text { Nord- } \\
\text { soudanienne }\end{array}$} & Gaya & Niger & 8,66 & 173,33 \\
\cline { 2 - 5 } & Tiogo & Burkina Faso & 2,14 & $4,2,80$ \\
\hline \multirow{2}{*}{$\begin{array}{l}\text { Sud- } \\
\text { soudanienne }\end{array}$} & Laba & Burkina Faso & 1,70 & 34,04 \\
\cline { 2 - 5 } & Cassou & Burkina Faso & 3,10 & 63,24 \\
\hline $\begin{array}{l}\text { Soudano- } \\
\text { guinéenne }\end{array}$ & Comoé-Léraba & Burkina Faso & 1,13 & 22,60 \\
\hline
\end{tabular}

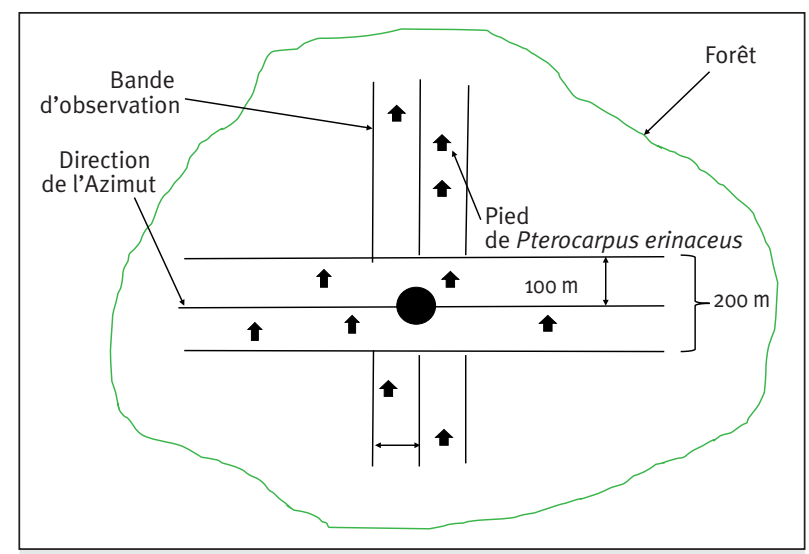

Figure 2.

Schéma du dispositif de collecte des données au sein des peuplements de Pterocarpus erinaceus.

\section{Analyse des données}

\section{Caractéristiques structurales}

Une analyse de variance a été effectuée pour comparer les paramètres dendrométriques (diamètre, hauteur totale, hauteur de fût) des peuplements de $P$. erinaceus dans les différentes zones pédoclimatiques échantillonnées. Les données qui ne suivent pas une distribution normale selon le test de Ryan Joiner (Rondeux, 1999) ont été comparées à l'aide du test non paramétrique de Kruskall Wallis. Les logiciels R 2.15.3 (R Development Core Team, 2010) et Minitab 16 ont été utilisés.

À partir des données collectées sur le terrain, la densité $(N)$, la surface terrière $(G)$ et la hauteur moyenne de Lorey (H) du peuplement ont été calculées afin de comparer les peuplements.

La densité $N$ est le nombre moyen d'individus par hectare :

$\mathrm{N}=\frac{\mathrm{n}}{\mathrm{s}}$

(équation 1)

avec $n$ le nombre d'individus et $s$ la superficie en hectares.

La surface terrière $\mathrm{G}$, exprimée en $\mathrm{m}^{2} /$ ha, est la densité surfacique de la somme des sections transversales des individus :

$\mathrm{G}=\frac{\pi}{4 \mathrm{~S}} \sum_{\mathrm{i}=1}^{\pi} \mathrm{di}^{2}$

(équation 2)

avec di le diamètre de l'individu $i$, exprimé en mètres, et $s$ la superficie couverte, exprimée en hectares.

La hauteur moyenne de Lorey $\left(\mathrm{H}_{\mathrm{L}}\right)$, ou hauteur de l'arbre moyen, est la moyenne des hauteurs des individus pondérées par leur surface terrière (Philip, 2002) :

$H L=\frac{\sum_{i=1}^{\pi} \text { gi hi }}{\sum_{i=1}^{\pi} \text { gi }} \quad$ avec $g i=\frac{\pi}{4} \mathrm{di}^{2}$

(équation 3)

avec gi la surface terrière de l'individus $i$, hi la hauteur de l'individu $i$ (exprimée en mètres) et di le diamètre de l'individu $i$ (en cm).

Les valeurs moyennes des différents paramètres dendrométriques mesurés (hauteur, diamètre, hauteur de fût) et des autres paramètres calculés (densité, surface terrière, hauteur de Lorey) ont été utilisées pour une analyse en composantes principales (ACP) visant à caractériser et discriminer les sites en fonction des zones pédoclimatiques.

\section{Structure démographique}

À partir d'un seuil de $5 \mathrm{~cm}$, des classes de diamètre d'amplitude $5 \mathrm{~cm}$ ont été constituées. Les classes de hauteur ont été définies à partir d'un seuil de $1 \mathrm{~m}$, avec une amplitude d'également $1 \mathrm{~m}$. Ces classes ont permis d'établir des histogrammes de distribution des classes de diamètre et de hauteur.

Un test d'ajustement à la distribution théorique de Weibull (Rondeux, 1999) a été effectué à l'aide du logiciel Minitab 16. La distribution théorique de Weibull à trois paramètres (de position $a$, d'échelle ou taille $b$ et de forme $c$ ) a été utilisée pour caractériser la structure des peuplements, à la faveur de sa souplesse d'emploi et de la grande variabilité 
de formes de distribution qu'elle produit. Le paramètre $a$ correspond à la valeur seuil, c'est-à-dire à la plus petite valeur de diamètre (respectivement de hauteur) retenue pour la constitution des histogrammes. Le paramètre $b$ est lié à la valeur centrale de la distribution des classes de diamètre et de hauteur. Enfin, le paramètre $c$ est lié à la structure observée et, selon sa valeur, conduit la distribution de Weibull à prendre plusieurs formes.

Sa fonction de densité de probabilité $f(x)$ se présente sous la forme suivante (Johnson et Kotz, 1970) :

$$
f(x)=\frac{c}{b}\left(\frac{x-a}{b}\right)^{c-1} \quad \exp \left[-\left(\frac{x-a}{b}\right)^{c}\right] \quad \text { (équation 4) }
$$

avec $x$ le diamètre $(\mathrm{cm})$, la circonférence $(\mathrm{cm})$ ou la hauteur (m) des arbres, $f(x)$ sa valeur de densité de probabilité générée à partir des centres des classes de diamètre ou de hauteur et des paramètres $a, b$ et $c$.

Pour vérifier la significativité de l'ajustement selon l'hypothèse nulle d'égalité entre la fréquence observée d'une classe de diamètre (respectivement de hauteur) considérée et la fréquence théorique attendue selon la fonction de Weibull (Agresti, 2010), une analyse log-linéaire, méthode itérative d'analyse de la variance du logarithme des densités des classes, a été effectuée.

\section{Résultats}

\section{Paramètres structuraux des peuplements}

De manière générale, la densité de $P$. erinaceus varie significativement $(P<0,001)$ selon le gradient d'aridité climatique, avec une valeur de 1,2 $\pm 0,7$ individu/ha dans la zone sahélo-soudanienne, 2,6 \pm 2,2 individu/ha dans la zone nord-soudanienne, 6,1 \pm 4,6 individu/ha dans la zone sud-soudanienne et 15,0 $\pm 1,1$ individu/ha dans la zone soudano-guinéenne. Cette densité diffère d'une formation à une autre au sein d'une même zone pédoclimatique. Ainsi, dans la zone nord-soudanienne, la densité est de 5,07 $\pm 2,7$ individus/ha dans la forêt de Saponé, et de 0,6 \pm 0,9 pieds/ ha seulement dans la forêt de Gaya au Niger. Dans la zone sud-soudanienne, la forêt de Cassou compte 9,4 \pm 4,9 individus/ha, pour 2,8 $\pm 2,4$ pieds/ha seulement dans la forêt de Laba (tableau II).

Le diamètre moyen de $P$. erinaceus est négativement corrélé au gradient climatique, avec les valeurs les plus élevées au sein de la zone sahélo-soudanienne $(47,2 \pm 15,6 \mathrm{~cm})$ et de la zone nord-soudanienne $(35,1 \pm 11,8 \mathrm{~cm})$. Les peuplements dans la zone sud-soudanienne sont caractérisés par un diamètre moyen de $29,5 \pm 13,9 \mathrm{~cm}$ contre $24,5 \pm 17,6 \mathrm{~cm}$ en zone soudano-guinéenne. Cette différence significative des valeurs de diamètre entre les zones $(P<0,001)$ s'observe également entre les formations d'une même zone. Ainsi, en zone sahélo-soudanienne, les peuplements de $P$. erinaceus du Parc $W$ présentent un diamètre moyen plus élevé que ceux de Tamou. II en est de même entre les forêts de la zone nord-soudanienne et de la zone sudsoudanienne. Le plus faible diamètre est observé dans la forêt de Comoé-Léraba, en zone soudano-guinéenne. Cette même forêt se caractérise cependant par les plus grandes valeurs de hauteur de fût, de surface terrière et de hauteur de Lorey, comparativement aux autres forêts investiguées (tableau II).

L'analyse en composantes principales (ACP) réalisée sur les valeurs moyennes des paramètres dendrométriques obtenus révèle que les deux premiers facteurs de l'analyse portent plus de $84,7 \%$ de la variance expliquée. L'axe 1 concentre le maximum de cette variance $(51,6 \%)$ et traduit un gradient pédoclimatique majoritairement responsable de la variation observée entre les paramètres dendrométriques des différents sites. Le plan factoriel principal révèle que le peuplement de la forêt de Comoé-Léraba située en zone soudano-guinéenne présente une densité élevée et une grande surface terrière de $P$. erinaceus, mais reste marqué par un faible diamètre moyen. La zone est également caractérisée par une grande hauteur de Lorey et un rapport élevé

Tableau II.

Caractéristiques dendrométriques.

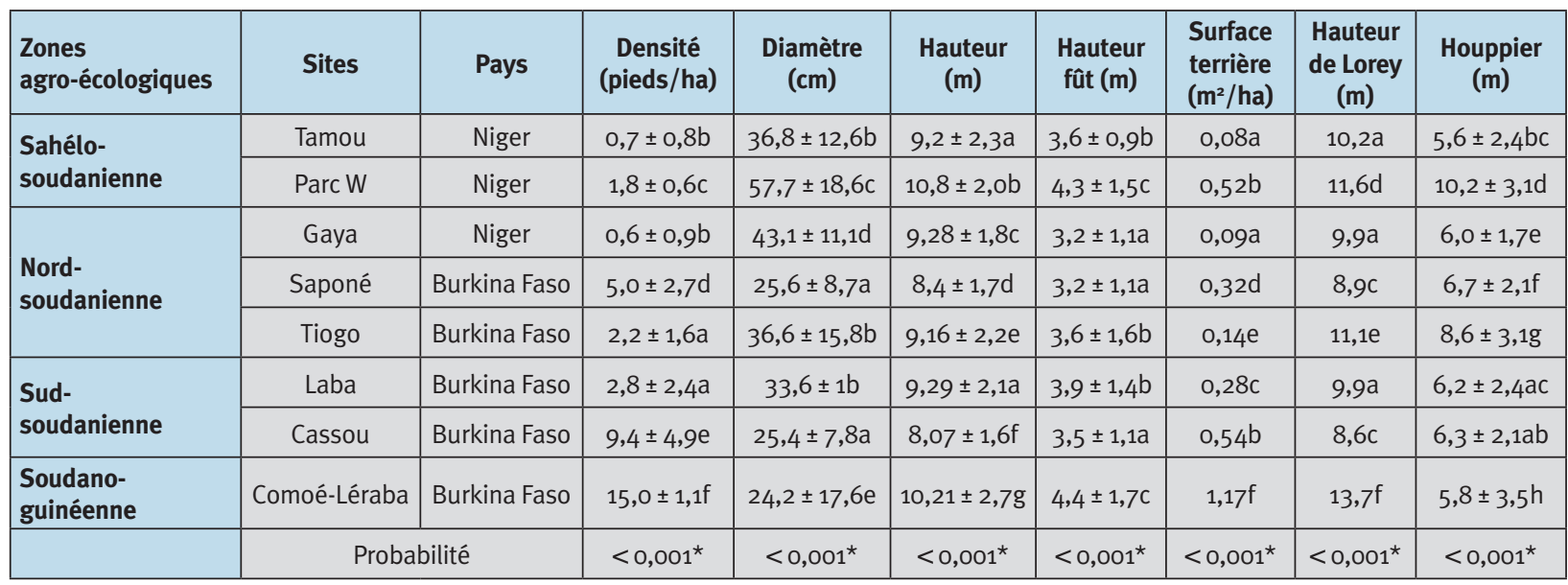

Dans une même colonne, les cases ayant les mêmes lettres n’ont pas des différences significatives ; * : différences significatives selon l’Anova. 
entre la hauteur de fût et la hauteur totale $(\mathrm{Hf} / \mathrm{Ht})$. Les sites de la zone sahélo-soudanienne sont caractérisés par un grand diamètre moyen, ce qui traduit un grand paramètre de forme $c$ de la distribution théorique de Weibull (figure 3).

Les sites présentant un diamètre moyen élevé sont également caractérisés par un grand paramètre de forme $c$ de la distribution théorique de Weibull. Dans les zones moins arrosées, les peuplements ne présentent que des individus de gros diamètre (tableau II). La fréquence des jeunes individus est plus élevée dans les zones les plus arrosées, et la prise en compte de ces individus dans l'établissement des valeurs moyennes des paramètres dendrométriques induit de fait une réduction du diamètre moyen. Bien que les individus les plus gros soient observés sur ces sites, les individus les plus faibles en diamètre y sont également rencontrés. Plus le paramètre de forme $c$ de la distribution théorique de Weibull des classes de diamètre est faible, et plus le peuplement présente une prédominance des individus jeunes ou de faible diamètre.

\section{La pression sur les peuplements}

Les individus de $P$. erinaceus sont soumis à diverses pressions humaines telles que l'écorçage et l'émondage dans toutes les forêts de toutes les zones, sauf dans les forêts protégées telles que la forêt de Comoé-Léraba et du Parc régional du W. L'intensité d'écorçage est la plus critique dans la forêt de Gaya au Niger, suivie de la forêt de Saponé située en zone nord-soudanienne au Burkina Faso. Les plus faibles proportions d'individus écorcés sont observées au sein des zones sud-soudanienne et soudano-guinéenne. L'espèce est victime de l'émondage exercé suivant diverses intensités selon les zones. Ainsi, la forêt de Gaya se caractérise par plus de $97,1 \%$ des individus émondés à plus de $50 \%$ de leur houppier. Les plus faibles pourcentages d'individus émondés sont observés dans les forêts de Saponé et de Tiogo (tableau III).

\section{Type de sol et distribution de Pterocarpus erinaceus}

Pterocarpus erinaceus est majoritairement observé sur les sols limono-argileux en zones sahélo-soudanienne et nord-soudanienne. En zones sud-soudanienne et soudanoguinéenne, il est beaucoup plus fréquemment observé sur sols sablo-limoneux. Sa présence sur sols latéritiques a été relevée uniquement dans la réserve de faune de Tamou, en zone sahélo-soudanienne (tableau III).

\section{Structure diamétrique des peuplements naturels de Pterocarpus erinaceus}

La distribution des classes de diamètre observées en zone sahélo-soudanienne, zone la moins arrosée, montre une prédominance des individus de classe de diamètre

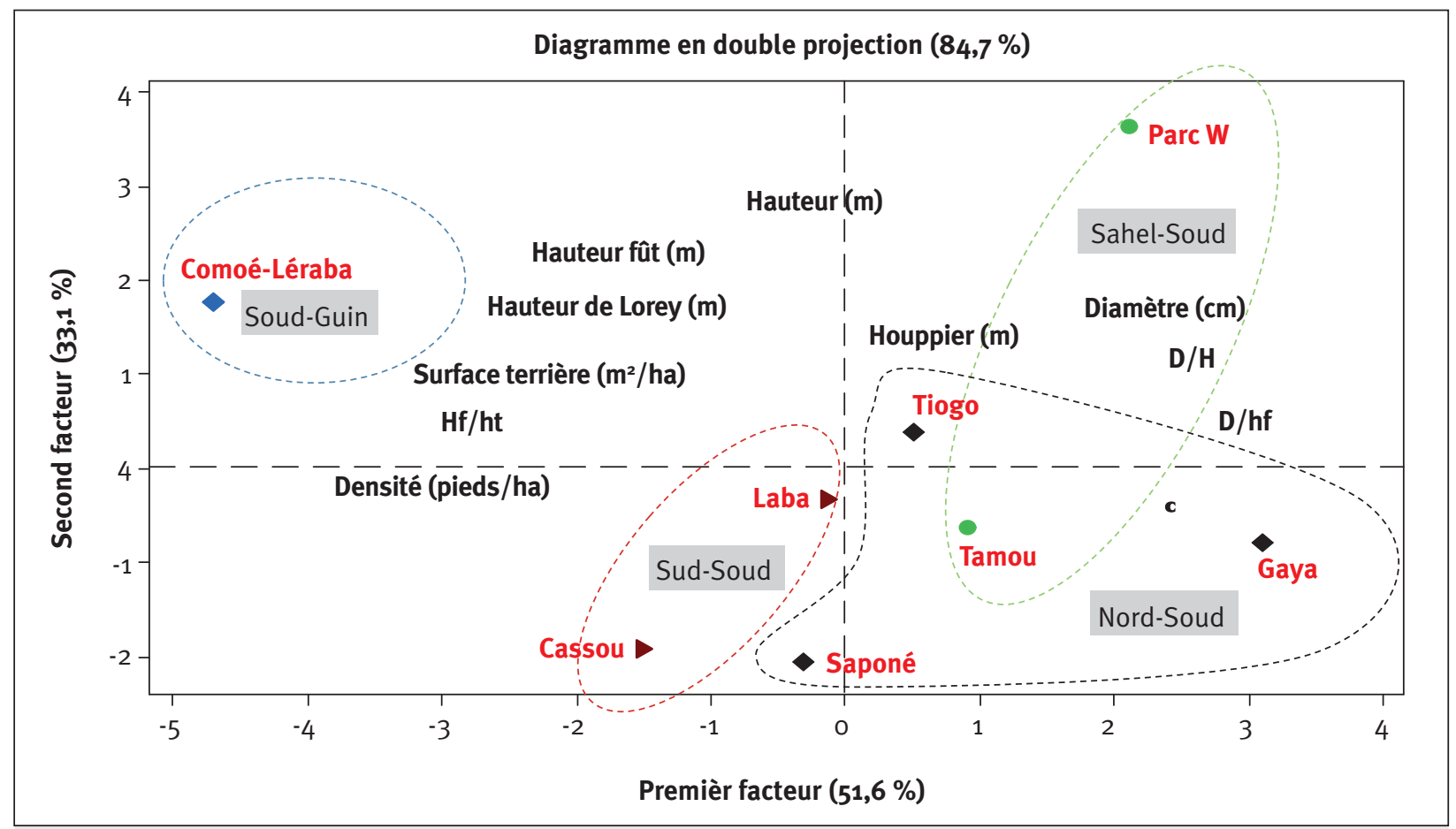

Figure 3.

Plan factoriel principal de l'ACP des caractéristiques dendrométriques des populations de Pterocarpus erinaceus. Hf : hauteur fût ; ht : hauteur totale ; D : diamètre ; $\mathbf{c}$ : paramètre de forme de la distribution de Weibull ; Soud-Guin : soudano-guinéenne ; Sahel-Soud : sahélo-soudanienne ; Nord-Soud : nord-soudanienne ; Sud-Soud : sud-soudanienne. 
Tableau III.

Intensité d'exploitation et type de sol.

\begin{tabular}{|c|c|c|c|c|c|c|c|c|c|c|c|}
\hline \multirow{2}{*}{$\begin{array}{l}\text { Zones agro- } \\
\text { écologiques }\end{array}$} & \multirow{2}{*}{ Sites } & \multirow{2}{*}{$\begin{array}{l}\text { Écorçage } \\
(\%)\end{array}$} & \multirow{2}{*}{$\begin{array}{c}\text { Émondage } \\
\text { (\%) }\end{array}$} & \multicolumn{2}{|c|}{$\begin{array}{c}\text { Intensité } \\
\text { de l'émondage }\end{array}$} & \multicolumn{6}{|c|}{ Texture du sol (\%) } \\
\hline & & & & $25 \%-50 \%$ & $>50 \%$ & Limoneux & $\begin{array}{l}\text { Limono- } \\
\text { argileux }\end{array}$ & Sableux & $\begin{array}{c}\text { Sablo- } \\
\text { limoneux }\end{array}$ & Latérite & Argileux \\
\hline \multirow{2}{*}{$\begin{array}{l}\text { Sahélo- } \\
\text { soudanienne }\end{array}$} & Tamou & 24,3 & 85,2 & 39,37 & 60,6 & 0 & 81,7 & 0 & 0 & 18,2 & 0 \\
\hline & PW & 0 & 0 & 0 & 0 & 0 & 68,3 & 0 & 31,7 & 0 & 0 \\
\hline \multirow{2}{*}{$\begin{array}{l}\text { Nord- } \\
\text { soudanienne }\end{array}$} & Saponé & 80,9 & 54,9 & 53,6 & 46,3 & 15 & 50,7 & 0 & 35,3 & 0 & 0 \\
\hline & Tiogo & 5,2 & 52,2 & 72,5 & 27,5 & 0 & 44,0 & 0 & 55,9 & 0 & 0 \\
\hline \multirow{2}{*}{$\begin{array}{l}\text { Sud- } \\
\text { soudanienne }\end{array}$} & Laba & 7,2 & 81,2 & 31,2 & 68,7 & 19,3 & 51,0 & 0 & 21,9 & 0 & 7,1 \\
\hline & Cassou & 2,5 & 70,5 & 31,5 & 68,4 & 16,5 & 23,5 & 19 & 31,7 & 0 & 0 \\
\hline $\begin{array}{l}\text { Soudano- } \\
\text { guinéenne }\end{array}$ & $\begin{array}{l}\text { Comoé- } \\
\text { Léraba }\end{array}$ & 0 & 0 & 0 & 0 & 0 & 0 & 0,6 & 95,8 & 0 & 3,5 \\
\hline
\end{tabular}

compris entre 30 et $55 \mathrm{~cm}$, soit $49,1 \%$ de l'effectif total contre $60,1 \%$ et $44,2 \%$ pour les peuplements respectifs de Tamou et du Parc W. En zone nord-soudanienne, la distribution des classes de diamètre montre une prédominance $(62,0 \%)$ des individus de la classe de diamètre $20-40 \mathrm{~cm}$, avec des valeurs de 70,0 \% et 29,8\% dans les peuplements respectifs de Saponé et de Gaya. La zone sud-soudanienne est caractérisée par une prédominance $(72,0 \%)$ des individus de diamètre compris entre 15 et $35 \mathrm{~cm}$, avec des valeurs de $84,8 \%$ et $48,6 \%$ dans les peuplements respectifs de Cassou et de Laba.

Dans toutes les zones investiguées, hormis la zone soudano-guinéenne, les individus de petit diamètre sont pratiquement absents, et les peuplements de $P$. erinaceus restent dominés par des individus de gros diamètre. L'analyse loglinéaire montre que l'ensemble de ces structures s'ajuste aux différentes distributions théoriques de Weibull avec $\mathrm{P}<0,05$.

La zone soudano-guinéenne est la seule zone qui présente une structure en diamètre de $P$. erinaceus avec prédominance d'individus de petit diamètre. Cette distribution des classes de diamètre observées s'ajuste $(P<0,05)$ à une distribution théorique de Weibull avec un paramètre de forme $c=1,21$, caractéristique des peuplements à prédominance d'individus jeunes ou de faible diamètre (figure 4).

\section{Structure en hauteur}

La distribution des classes de hauteur révèle une prédominance d'individus moyens. En zone sahélo-soudanienne, les individus de classe de hauteur 8-12 m sont les plus représentés (69,8\%), avec 72,9\% pour le Parc W et 62,2 \% pour le peuplement de Tamou. En zone nord-soudanienne, la structure est dominée par des individus de classe de hauteur 6-10 m (soit 74,3\%), cette valeur s'élevant à 47,8 \% seulement dans les peuplements de Tiogo, 76,3 \% à Gaya, et $83,4 \%$ dans les peuplements de Saponé. La zone sudsoudanienne est également caractérisée par une prédominance $(81,0 \%)$ des individus de classe de hauteur 6-10 m, avec $88,1 \%$ à Cassou et $69,1 \%$ pour le peuplement Laba.
La zone soudano-guinéenne est dominée (72,8 \%) par des classes de hauteur comprise entre 7 et $12 \mathrm{~m}$, ce qui reflètent les peuplements de la réserve de faune de Comoé-Léraba. Toutes les zones pédoclimatiques, hormis la zone soudanoguinéenne, sont caractérisées par un paramètre de forme de la distribution théorique de Weibull supérieur à 4, ce qui est caractéristique de peuplements où les individus moyens prédominent. L'analyse log-linéaire montre que toutes les structures des hauteurs observées s'ajustent avec la distribution de Weibull (P>0,05) (figure 5).

\section{Discussion}

\section{Les attributs structuraux}

L'analyse des caractéristiques dendrométriques des peuplements de $P$. erinaceus suivant un gradient climatique révèle une relation positive entre la densité des peuplements, la surface terrière et l'humidité du climat : la densité moyenne et la surface terrière y sont plus élevées en zones plus humides. La distribution de $P$. erinaceus serait ainsi fortement liée au régime pluviométrique.

Cette étude a également permis de montrer que la densité de $P$. erinaceus dépendait du statut de protection de la forêt. En effet, la forêt de Gaya en zone nord-soudanienne au Niger est une forêt exploitée et présente une densité relativement inférieure à celle du Parc W, qui relève de la zone sahélienne mais est intégralement protégé. Il en est de même pour la forêt de Saponé en zone nord-soudanienne, qui bénéficie d'une protection intégrale. Cette forêt présente une densité d'individus supérieure à celle de la forêt de Laba, exploitée en zone sud-soudanienne. Ces résultats confirment ceux obtenus par Ouédraogo et al. (2006) selon lesquels la densité de $P$. erinaceus est de 5,5 \pm 0,6 individus/ha dans la zone nordsoudanienne et de 9,8 \pm 3,3 individus/ha dans la zone sudsoudanienne. Ces densités sont en revanche inférieures à celles relevées par Glele et al. (2008) dans les zones de transition soudano-guinéenne du Bénin, elles-mêmes déjà 


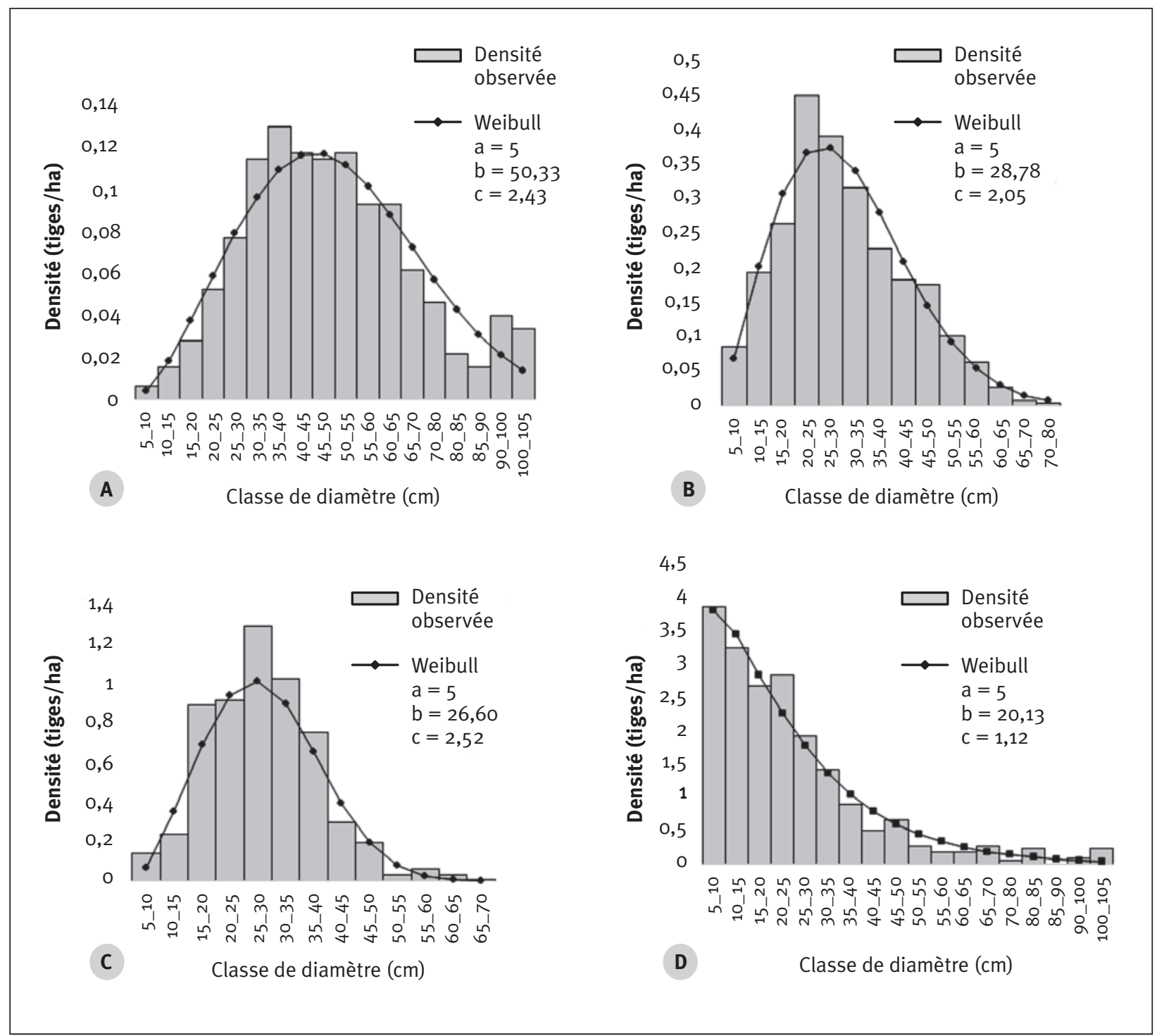

Figure 4.

Structure en diamètre de Pterocarpus erinaceus des différentes zones d'étude : zones sahélo-soudanienne (A), nord-soudanienne (B), sud-soudanienne et soudano-guinéenne (D).

inférieures à celles trouvées par Adjonou et al. (2010) dans la même zone pédoclimatique au Togo. Ces différences pourraient s'expliquer par la différence des conditions écologiques qui règnent dans ces zones mais aussi par la différence de l'approche adoptée dans la collecte des données. En effet, l'approche par transect en bande utilisée dans le cadre du présent travail permet de couvrir une surface plus grande et par conséquent d'accroître l'effort d'échantillonnage.

Le diamètre moyen élevé observé en zones sahélosoudanienne et nord-soudanienne résulte de la prédominance d'individus âgés. Le plus faible diamètre moyen relevé en zones sud-soudanienne et soudano-guinéenne s'explique par la fréquence élevée des individus jeunes de faible diamètre. Par contre, la dominance des individus de gros diamètre observés en zone sahélo-soudanienne pourrait être liée aux facteurs climatiques (diminution de la pluviométrie, concentration des pluies sur une courte période de l'année) conjugués aux facteurs anthropiques qui constituent les principaux obstacles à la régénération. Les individus de petit diamètre sont moins représentés en raison d'un déficit de recrutement. En effet, $P$. erinaceus dissémine ses fruits au mois d'avril, peu avant la saison des pluies (Mahamane et al., 2007). Ceux-ci tombent au sol et, dès les premières pluies, s'imbibent grâce au revêtement pileux du péricarpe, ce qui induit une forte réponse germinative (Arbonnier, 2004). Mais en zone sahélienne, un mois peut s'écouler entre la première et la deuxième pluie, la majorité des graines germées dépérissant alors. 


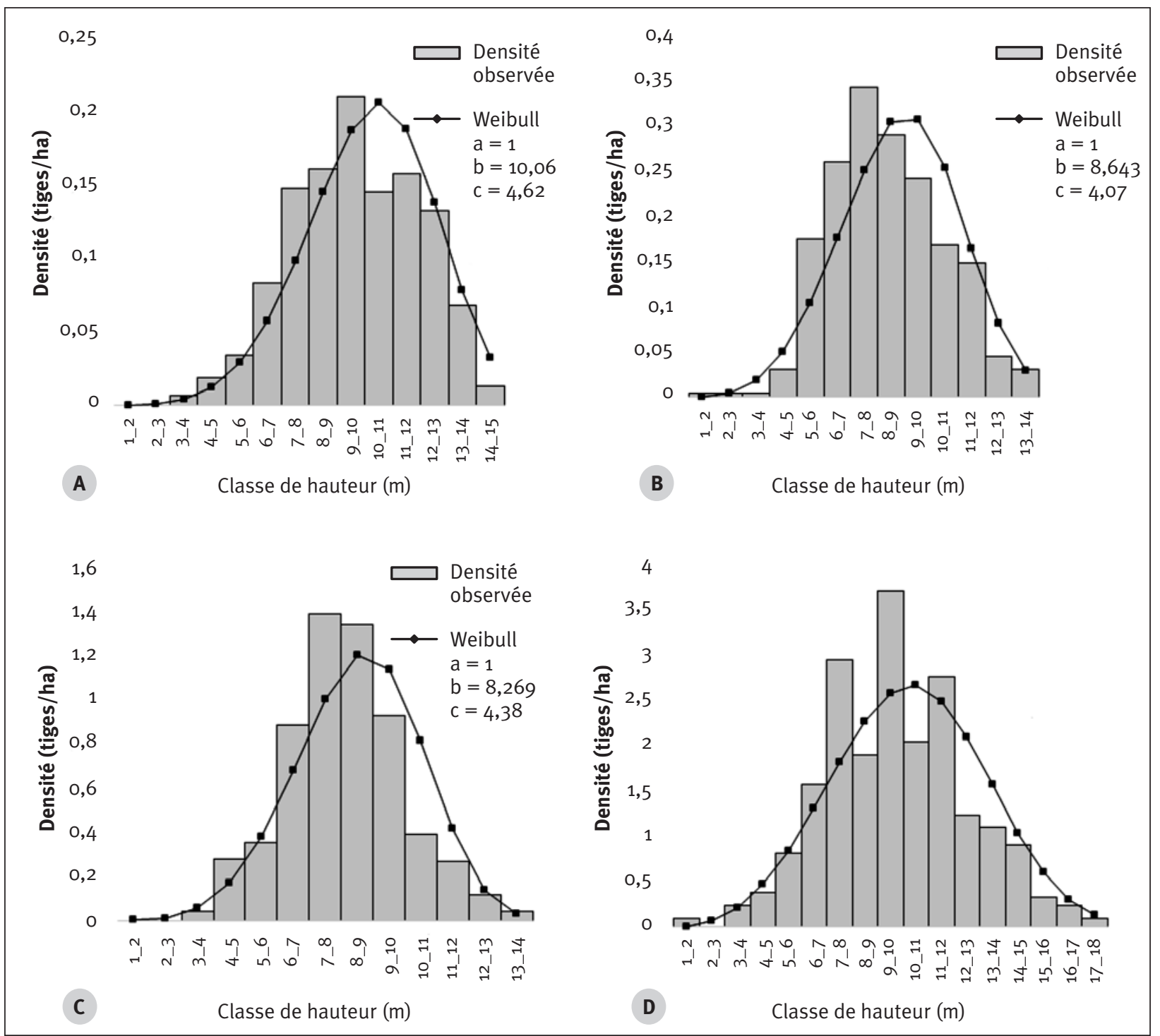

Figure 5.

Structure en hauteur de Pterocarpus erinaceus dans les différentes zones d'étude : zones sahélo-soudanienne (A), nord-soudanienne (B), sud-soudanienne (C) et soudano-guinéenne (D).

Les gros diamètres observés dans la forêt de Gaya et la réserve de faune de Tamou en zones sahélo-soudanienne et nord-soudanienne, s'agissant de forêts à vocation pastorale, rendent compte du constant émondage exercé par les bergers. La mutilation des branches au fil des années par les paysans limite la croissance en hauteur, favorisant plutôt la croissance en diamètre (De Caluwé, 2005 ; Assogbadjo, 2006). Ceci pourrait expliquer la faible élongation de la hauteur du fût au profit de la croissance latérale observée en zones sahélo-soudanienne et nord-soudanienne. Par contre, en zones sud-soudanienne et soudano-guinéenne, la hauteur du fût reste relativement plus élevée si l'on se réfère à la hauteur totale, ce qui résulte peut-être d'une densité plus élevée.

\section{Évolution des structures en diamètre et en hauteur}

La structure en diamètre des peuplements naturels de $P$. erinaceus révèle globalement une grande représentativité des individus âgés de gros diamètre, hormis la forêt de ComoéLéraba située en zone soudano-guinéenne. Cette exception s'explique par les caractéristiques écologiques et climatiques de cette zone qui se présente comme la plus arrosée de nos zones d'étude. Les individus de diamètre supérieur à $1 \mathrm{~m}$ n'ont été observés que dans le Parc W du Niger situé dans la zone sahélo-soudanienne et la réserve partielle de faune de ComoéLéraba en zone soudano-guinéenne, qui sont toutes des aires 
intégralement protégées. Celles-ci jouent non seulement un rôle de conservation des écosystèmes, mais offrent aussi les meilleures conditions à la croissance de certaines espèces (Goreaud, 2000). Dans la zone soudanienne, bien que les conditions d'humidité soient favorables, $P$. erinaceus est confronté à un problème de régénération. L'absence d'individus jeunes, particulièrement dans les aires protégées tel le Parc $W$ où la pression anthropique est absente, pourrait être liée aux facteurs climatiques. Sylla et al. (2002) suggèrent que, malgré le statut protégé de l'espèce dans les forêts classées et parcs nationaux du Sénégal, des peuplements ont disparu sous l'effet de la sécheresse. Plusieurs auteurs ont mis en évidence la bonne capacité de germination des graines de $P$. erinaceus et l'incapacité des plantules à franchir les différents stades de développement jusqu'à l'âge adulte à cause des agents perturbateurs tels que les facteurs climatiques, l'herbivorie, les feux de végétation et le défrichement (Bationo et al., 2001 ; Ouédraogo et al., 2006 ; Nacoulma, 2012).

\section{Pressions anthropiques sur l'espèce}

$P$. erinaceus fait l'objet d'une exploitation fourragère par les éleveurs et d'un écorçage à des fins médicinales. En zone sahélo-soudanienne, plus de 60,6\% des individus de $P$. erinaceus ont leur houppier émondé à plus de $50 \%$. Ce taux passe à 97,1 \% dans la forêt de Gaya, exploitée surtout en saison sèche au moment où le fourrage herbacé est rare. $P$. erinaceus est une espèce prisée pour son fourrage aérien (Sokpon et al., 2006 ; Ouédraogo et al., 2006). La récolte des feuilles et des fruits est généralement effectuée en fin de saison sèche, période durant laquelle l'herbe est rare. Les feuilles de $P$. erinaceus jouent un rôle important dans la digestion de la cellulose de l'herbe sèche. $P$. erinaceus fournit le fourrage aérien le plus vendu à Bobo-Dioulasso au Burkina Faso, et à Gaya au Niger (Sanou et al., 2011 ). En 1990, plus de 1400 tonnes de feuillage frais, dont $78 \%$ provenant de $P$. erinaceus, ont été vendues à Bamako (Mali) comme aliment pour les petits ruminants. Cette espèce autrefois dominante dans les forêts périurbaines de l'Ouest du Burkina Faso y a aujourd'hui disparu (Fournier et al., 2001).

$P$. erinaceus est une plante médicinale utilisée par les populations contre des maladies virales dans les régions centrales du Burkina Faso. Son écorce sert pour le traitement des affections inflammatoires, ulcères gastriques, rhumatismes et dermatoses (Ouédraogo et al., 2012). Ceci explique la fréquence de l'écorçage dans les zones soudanienne et sahélo-soudanienne, notamment dans les forêts proches des grands centres urbains comme la forêt de Gaya située à $7 \mathrm{~km}$ de la ville de Gaya au Niger et la forêt de Saponé située à $30 \mathrm{~km}$ de Ouagadougou.

\section{Distribution de l'espèce selon le type de sol}

Le type de sol semble jouer un rôle majeur dans la distribution de $P$. erinaceus (Grimaldi et Riera, 2001). La bonne distribution de l'espèce sur sol limoneux-argileux dans les zones les moins arrosées peut s'expliquer par une bonne capacité de rétention en eau, malgré à un régime pluviométrique pourtant faible. Les sols sableux-limoneux favorisent au contraire l'infiltration des eaux au détriment de la végétation. En revanche, dans les zones les plus arrosées, l'espèce est capable de s'épanouir pleinement, même sur de tels sols sableux-limoneux.

$P$. erinaceus est observé sur tous types de sols, mais il préfère les sols acides à neutres, légers à moyens, drainant librement (Arbonnier, 2004). Selon Goreaud (2000), les différents types de sols peuvent correspondre à différentes profondeurs d'enracinement des plantes. Toutefois, $P$. erinaceus a également été observé sur les plateaux de la réserve de faune de Tamou en zone sahélo-soudanienne, s'agissant de sols non profonds ne dépassant pas 30 à 40 cm (Karim et al., 2003).

\section{Conclusion}

L'étude des caractéristiques dendrométriques de Pterocarpus erinaceus suivant les zones pédoclimatiques a montré que la densité augmentait selon le gradient d'aridité nord-sud. L'espèce se régénère mieux dans les zones les plus arrosées. La zone sahélienne est caractérisée par un peuplement à dominance d'individus de gros diamètre. Les individus jeunes ou de faible diamètre y sont presque absents, ce qui traduit un manque de régénération de l'espèce, y compris dans le Parc W du Niger, aire intégralement protégée. En plus de la pression anthropique (émondage, écorçage), les facteurs climatiques contribuent donc au dépérissement des peuplements de $P$. erinaceus dans cette zone. L'étude a également montré que l'intensité de l'émondage à des fins d'affouragement était plus élevée dans les zones les moins arrosées, alors que l'intensité de l'écorçage était plus critique dans les forêts proches des grands centres urbains. Ces pratiques constituent une entrave au développement de l'espèce dans ces zones.

\section{Remerciements}

La Commission de l'UEMOA (Union économique et monétaire ouest-africaine) a financé cette recherche par l'intermédiaire du PAES (Programme d'appui à l'enseignement supérieur) dans ses pays membres.

\section{Références bibliographiques}

Adjonou K., Ali N., Kokutse A. D. Segla K. N., Kokou K., 2010. Étude de la dynamique des peuplements naturels de $P$. erinaceus Poir. (Fabaceae) surexploités au Togo. Bois et Forêts des Tropiques, 306 (1) : 33-43.

[En ligne] http://bft.cirad.fr/cd/BFT_306_45-55.pdf

Agresti A., 2010. Analysis of Ordinal Categorical Data. 2nd edition. New York, USA, Wiley, $424 \mathrm{p}$.

Ambouta K. M. K., 1984. Contribution à l'édaphologie de la brousse tigrée de l'Ouest Nigérien. Thèse, Université de Nancy I, France, 116 p. 
Arbonnier M., 2004. Trees, shrubs and lianas of West African dry zones. Cirad, Margraf, MNHN, 574 p.

Assogbadjo A. E., 2006. Importance socio-économique et étude de la variabilité écologique, morphologique, génétique et biochimique du baobab (Adansonia digitata L.) au Bénin. Thèse, Faculté des sciences en bio-ingénierie, Université de Gand, Belgique, 213 p.

Bationo B. A., Ouédraogo S. J., Guinko S., 2001. Longévité des grains et contraintes à la survie des plantules d'Afzelia africana $\mathrm{Sm}$. dans une savane boisée du Burkina Faso. Annals of Forest Science, $58: 69-75$.

De Caluwé E., 2005. Etnobotanie, morfologische variabiliteit en natuurlijke regeneratie van Adansonia digitata in Atacora (Benin). Université de Gand, Belgique, 189 p.

Drabo I., Ilboudo F., Tallet B., Quesnel A., Marchal J.-Y. (éds), 2003. Dynamique des populations, disponibilités en terres et adaptation des régimes fonciers : le Burkina Faso, une étude de cas. Paris, France, CICRED, Rome, Italie, FAO, 114 p.

Fournier A., Floret C., Gnahoua G. M., 2001. Végétation des jachères et succession post-culturale en Afrique tropicale. In : Floret C., Pontanier R. (éds). La jachère en Afrique tropicale : rôles, aménagement, alternatives. Vol. 2 : De la jachère naturelle à la jachère améliorée : le point des connaissances. Actes du séminaire international, Dakar, 13-16 avril 1999. Paris, France, John Libbey Eurotext, 123-168.

Glele K. R. L., Sinsin B., Palm R., 2008. Étude dendrométrique de $P$. erinaceus Poir. des formations naturelles de la zone soudanienne au Bénin. Agronomie Africaine, 20 (3) : 245-255.

Goreaud F., 2000. Apports de l'analyse de la structure spatiale en forêt tempérée à l'étude et la modélisation des peuplements complexes. Thèse, Engref, option Sciences forestières, France.

Grimaldi M., Riera B., 2001. Geography and climate. In: Bongers F., Charles-Dominique P., Forget P.-M., Théry M. (eds). Nouragues. Dynamics and plant-animal interactions in a neotropical rainforest. Dordrecht, Pays-Bas, Kluwer Academic, 9-18.

Henry M., Picard N., Trotta C., Manlay R. J., Valentini R., Bernoux M., Saint-André L., 2011. Estimating tree biomass of sub-Saharan African forests: a review of available allometric equations. Silva Fennica, 45 (3): 477-569.

Johnson N. L., Kotz S., 1970. Distributions in Statistics: Continuous Univariate Distributions. New York, USA, John Wiley \& Sons, $756 \mathrm{p}$.

Karim S., Bationo B. A., Bellefontaine R., Ichaou A., 2003. Reboiser au moindre coût les zones semi-arides par marcottage naturel. In : Actes du XII ${ }^{\mathrm{e}}$ Congrès forestier mondial, Montréal, septembre 2003 : La forêt, source de vie. Des forêts pour la planète. FAO, Département des Forêts, Canada, Ministère des Ressources naturelles, Québec, Ministère des Ressources naturelles, de la Faune et des Parcs.
Mahamane A., Saadou M., Lejoly J., 2007. Phénologie de quelques espèces ligneuses du Parc national $d u$ « W »du Niger. Sécheresse, 1 (4) : 1-13.

Nacoulma B. M. I., 2012. Dynamique et stratégie de conservation de la végétation et de la phytodiversité du complexe écologique du Parc National du W du Burkina Faso. Thèse de doctorat, Université de Ouagadougou, Burkina Faso, 151 p.

Ouédraogo A., Adjima T., Hahn-Hadjali K., Guinko S., 2006. Diagnostic de l'état de dégradation des peuplements de quatre espèces ligneuses en zone soudanienne du Burkina Faso. Sécheresse, 17 (4) : 485-491.

Ouédraogo N., Sawadogo R. W., Tibiri A., Bayet C., Lompo M., Hay A. E., Koudou J., Dijoux M.-G., Guissou I. P., 2012. Pharmacological properties and related constituents of stem bark of Pterocarpus erinaceus Poir. (Fabaceae). Asian Pacific Journal of Tropical Medicine, 5 (1): 46-51.

Ouédraogo N., Sawadogo R. W., Tibiri A., Lompo M., Hay A. E., Koudou J., Dijoux M. G., Guissou I. P., 2011. Antioxidant anti-inflammatory and analgesic activities of aqueous extract from stem bark of Pterocarpus erinaceus Poir. (Fabaceae). Journal of Medicinal Plants Research, 5 (10): 2047-2053.

Philip S. M., 2002. Measuring Trees and Forests. 2nd edition. Londres, Royaume-Uni, CABI, $310 \mathrm{p}$.

R Development Core Team, 2010. R: A language and environment for statistical computing. Vienne, Autriche, R Foundation for Statistical Computing.

[Online] http://www.R-project.org

Rondeux J., 1999. La mesure des peuplements forestiers. Gembloux, Belgique, Les Presses agronomiques de Gembloux, $522 \mathrm{p}$.

Sanou K. F., Nacro S., Ouédraogo M., Ouédraogo S., Kaboré-Zoungrana C., 2011. La commercialisation de fourrages en zone urbaine de Bobo-Dioulasso (Burkina Faso) : pratiques marchandes et rentabilité économique. Cahiers Agricultures, 20 (6) : 487-493.

Sawadogo I., 2011. Ressources fourragères et représentations des éleveurs, évolution des pratiques pastorales en contexte d'aire protégée : cas du terroir de Kotchari à la périphérie de la réserve de biosphère du W au Burkina Faso. Thèse, Muséum national d'histoire naturelle, Paris, France, $345 \mathrm{p}$.

Sokpon N., Biaou S. N., Ouinsavi C., Hunhyet O., 2006. Bases techniques pour une gestion durable des forêts claires du Nord-Bénin : rotation, diamètre minimal d'exploitabilité et régénération. Bois et Forêts des Tropiques, 287 (1) : 45-57. [En ligne] http://bft.cirad.fr/cd/BFT_287_45-57.pdf

Sylla S. N., Samba R. T., Neyra M., Ndoye I., Giraud E., Willems A., De Lajudie P., Dreyfus B., 2002. Phenotypic and Genotypic Diversity of Rhizobia Nodulating $P$. erinaceus and $P$. lucens in Senegal. Systematic and Applied Microbiology, 25: 572-583. 